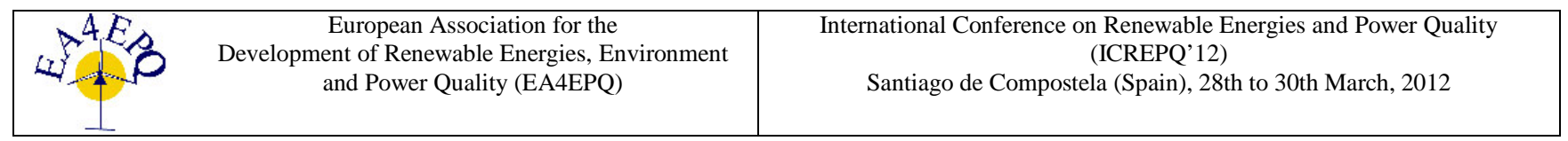

\title{
An extended EMC study of an electrical powertrain for transportation systems
}

\author{
B. Chand, J. Keghie, S. Dickmann \\ Faculty of Electrical Engineering, Helmut-Schmidt-University / University of the Federal Armed Forces Hamburg, \\ Germany \\ Phone number:+0049 40 6541-2395, e-mail: benian.chand@hsu-hh.de, jules.keghie@hsu-hh.de, \\ stefan.dickmann@hsu-hh.de
}

\begin{abstract}
The change from conventional to electric drives in transportation systems is forced by the shortage of gasoline and the environment protection. Electric drive systems are a challenge for the manufacturers. The electrical powertrain can not only be exposed to interferences, it may also emit interferences into components and communication cables in the vicinity. These can be a high risk for the drive system and for the passengers, when the electric drive is used for human transport. This paper presents an EMC study of an electrical powertrain for transportation systems. A brief summary of the interferences at the unshielded high-voltage cable (HV-cable) is given. The analysis of the influence of position variation of the communication cable to the unshielded HV-cables is studied. Different cheap shielding variants are analysed and compared to full shielded HV-cables. Furthermore, different optimizations have been added to the test setup.
\end{abstract}

\section{Key words}

EMC, Electrical Powertrain, unshielded/shielded HVCables, Power Converter, Bus Systems

\section{Introduction}

Almost every manufacturer of transportation systems extends his portfolio of products with electric drives $[1,2,3]$. The simplified structure of the electrical powertrain (see Figure 1) can not only be influenced from outside, it can also emit disturbances. These disturbances are critical, when important communication lines are influenced, which could put the drive or the safety of the passengers at risk. A simplified study was done in [4]. An extension to practical cases is done here. In addition, the interferences on the unshielded HV-cables are characterized. . In transportation systems the HV-cables are distributed over the whole vehicle. Therefore the position between $\mathrm{HV}$-cables and existing communication lines can vary. And these different positions yields to different coupling behaviours. For this reason, the position of the communication lines to the $\mathrm{HV}$-cables is an essential point in this study. Furthermore, the influence of variations of some parameters is studied. These parameters which are taken into consideration are the motor speed, the
PWM-frequency, the terminations and the load. An optimized control unit for the power converter is developed and built in this study, and optimizations on the measurement setup are done. Different communication cables are used for comparison.

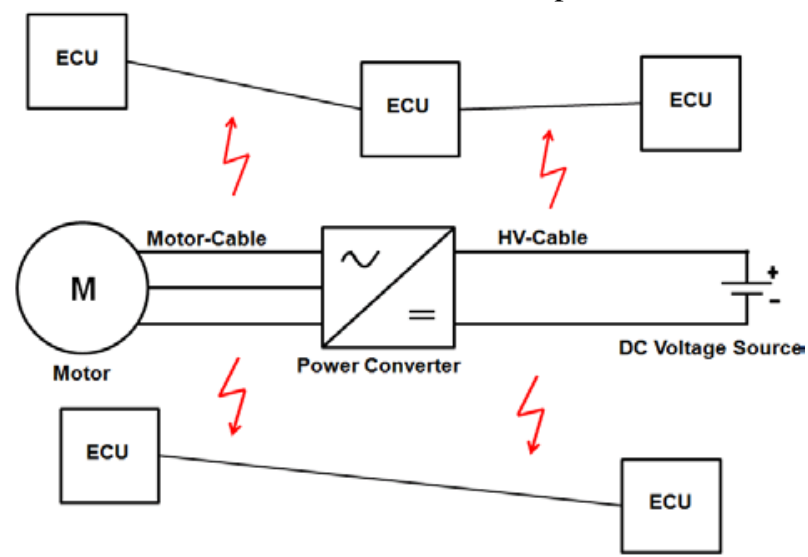

Fig. 1 Structure of the electrical powertrain and nearby Electronic Control Units (ECUs)

Since unshielded $\mathrm{HV}$-cables are less expensive in comparison to full shielded ones, a comparison of different shielding variants with unshielded and shielded $\mathrm{HV}$-cables is a main aspect in this study. For this reason the used components are shielded to avoid a disturbance of the analysis results. Also the influence of the chassis or other parts between the HV-cables and the communication cable is analyzed, using metal plates.

\section{Test setup}

In Figure 2 the measurement setup is shown. At first glance one should mean, the disturbances are only present on the motor cables between the motor and the power converter. This topic was presented in $[5,6]$. But this contribution shows, that disturbances are also present on the HV-cables at the DC side. Due to packaging reasons, the $\mathrm{HV}$-cables are distributed over the whole vehicle and therefore could influence all components in the vehicle. 


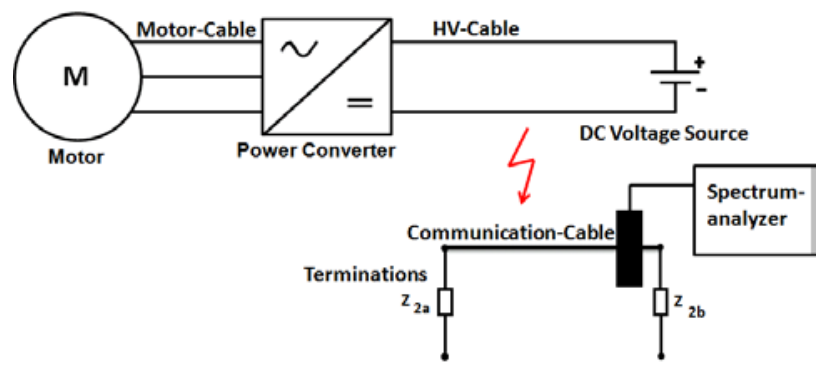

Fig. 2 Measurement setup

The motor cables in Figure 2 are kept very short or inserted into the housing of the motor, to minimize interferences from these cables.

The components of the test setup are a DC voltage source, a power converter, a control unit, a motor, a load, different cables and a spectrum analyzer. A detailed specification is given in [7]. The measurements are done using a signal analyzer under consideration of [8].

\section{Optimization of the test setup}

The measurements of the HV-cables show strong interferences, which come from the internal pulsed power supplies of the DC source. Since these unwanted interferences would have distorted the measurement results, a DC-filter (Figure 3) was constructed and further optimized with simulations for the required frequency range.

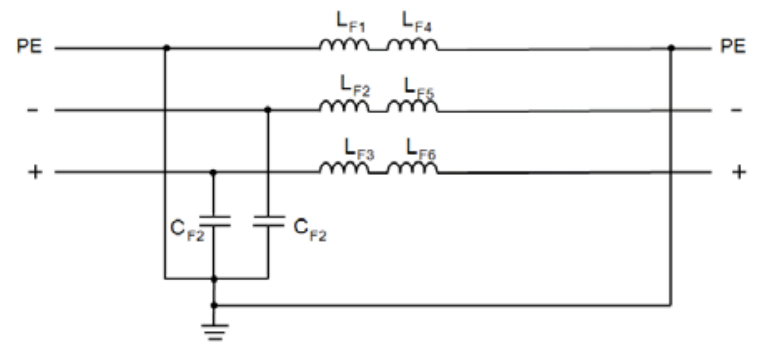

Fig. 3 Equivalent circuit of the DC-filter

The Figures 4 and 5 show the results with and without filter for both frequency ranges. One can see a good attenuation of interferences from the pulsed DC voltage source. The filter improved the signal from the DC source significantly.

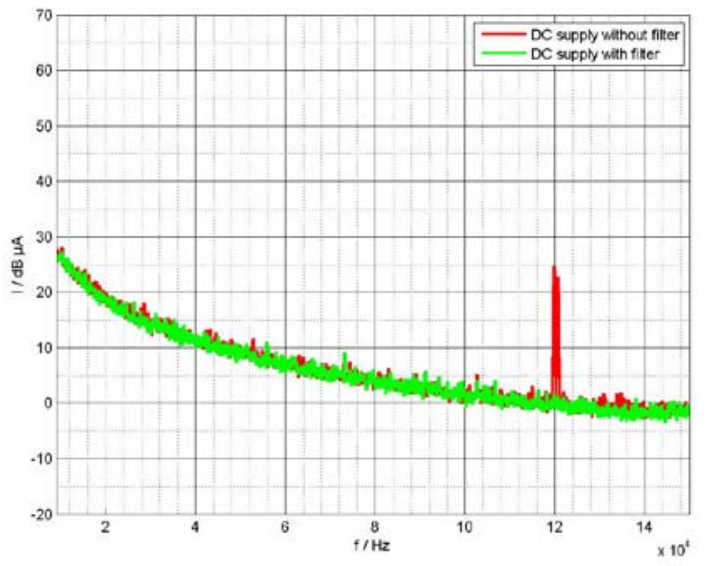

Fig. 4 Comparison of the results with filter and without filter, for the lower frequency range

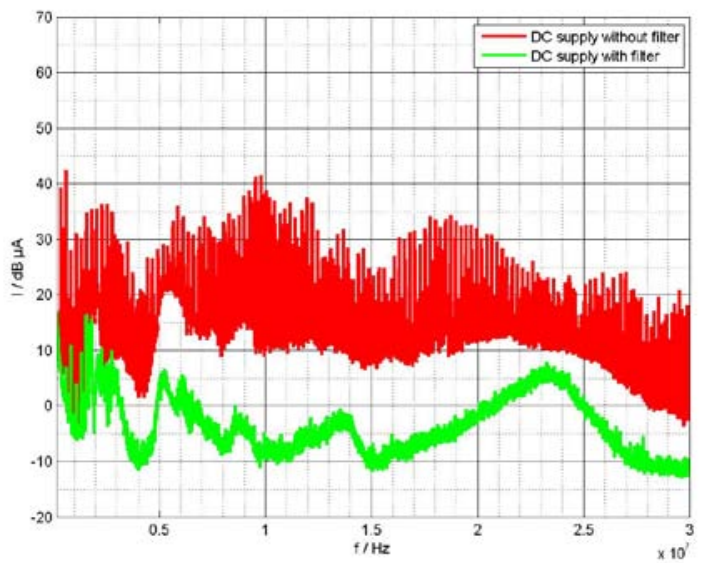

Fig. 5 Comparison of the results with filter and without filter, for the upper frequency range

\section{Measurements and evaluation}

\section{A. Optimized measurement setup}

The interconnection of the components described in Chapters 2 and 3 yields the following test setup in Fig. 6.

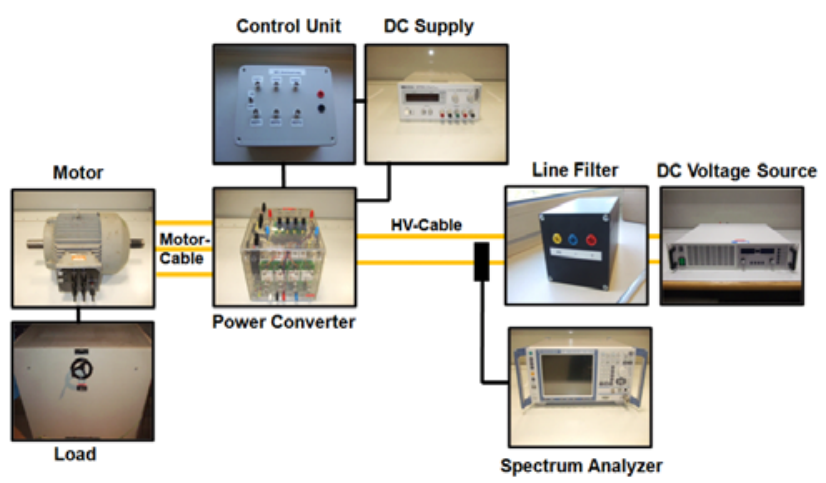

Fig. 6 Components of the whole test setup, connected to one possible measurement setup

The measurements are carried out in an Absorber Line Shielded Enclosure (ALSE) in order to avoid 
interferences from outside. The spectrum has been measured with a current measuring clamp in $\mathrm{dB} \mu \mathrm{V}$. Since the current through the cables is also of interest, one can calculate it using the frequency-dependent coupling factor $\mathrm{k}$ of the clamp (1).

$$
\frac{I}{d B \mu A}=\frac{U}{d B \mu V}+\frac{k}{d B \frac{1}{\Omega}}
$$

\section{B. Summary of the first part of the measurements \\ 1) Characterization of the unshielded $H V$-cables}

When measuring the current at various positions of both $\mathrm{HV}$-cables, no significant differences in the results of the measurements are seen. On the motor cables, the PWM frequency and its multiples can be seen clearly. In the higher frequency range the signal form and the magnitude change, like expected, when one compares the current of one cable to all three cables, due to the interaction of the currents on the three cables. The behaviour of the interferences on the $\mathrm{HV}$-cables is composed of the frequency dependent internal interferences of the components.

\section{2) Influence of the motor speed, the PWM frequency and the load of the engine}

The change of the motor speed at constant PWMfrequency, does not change the interference behaviour. This is because the resulting short-term disturbances are only transients and these are not considered here, since the frequency is measured under steady state condition. A change in the PWM-frequency at constant motor speed, however, shows a change of gradient. The higher the PWM frequency, the higher the interference currents and thus more resonances appear. In the lower frequency range, the PWM-frequencies and the multiples are clearly visible. An increase of the load, which results in an increase of the current, shows no significant differences in the frequency range of $9 \mathrm{kHz}-150 \mathrm{kHz}$. In the upper frequency range of $150 \mathrm{kHz}-30 \mathrm{MHz}$, the magnitude changes of course at a higher current, but also the signal form of the interference. The signal form of the interference changes, since for different current/voltage ratio the conducted interference of the components changes internally.

\section{3) Increasing the distance between the communication cable and the $\mathrm{HV}$-cables}

Increasing the distance of the measured cable parallel to the HV-cable yields as expected to decreasing magnitude. Three different values $(5 \mathrm{~cm}, 10 \mathrm{~cm}, 30 \mathrm{~cm}$ ) are used for the parallel distance between the cables. A decreasing of the parallel distance yields further to an increasing of the same ratio of $\mathrm{dB}$ of the interference current.
4) Change of the height profile of the measured cable with respect to the reference surface

Since the communication lines of transportation systems do not always have a fixed distance from the chassis, the influence on communication lines of different heights over a reference surface is studied. One side of the cable is set up $15 \mathrm{~cm}$ above the ground surface, the other is 5 $\mathrm{cm}$ above the ground plane. The result shows that such variation of the height profile of communication lines over a reference plane has a negligible influence for the interference.

5) Communication cable between the HV-cables, diagonally across the $\mathrm{HV}$-cables and perpendicular to the HV-cables

Furthermore, the influences of positioning the communication cable diagonally across the HV-cables, perpendicular to the HVcables and between the $\mathrm{HV}$ cables are compared. On the communication cable in the middle of the two HV-cables the most interferences can be seen. The inclined position of the measured cables causes a lower coupling of the interference current due to the coupling pattern of the E-and H-fields. The lowest coupling is thus obtained for vertically and centrally located communication cables. The change of the position of the communication cables yields up to $23 \mathrm{~dB}$ lower interferences.

\section{6) Influence of cable terminations at the communication cable}

Another important point is the investigation of the influence of the communication cable terminations. The terminations describe the input resistances in the ECUs in bus systems. It can be seen, that the terminations influence the standing wave patterns on the cables. There is a direct correlation between the choosen terminations and the current magnitude that can be measured. The differences can be up to $40 \mathrm{~dB}$, so it is necessary to consider the termination values well.

\section{7) Comparison of single wire and twisted-pair-cable}

Not only the single-wire measurements are done, also the twisted-pair-cable, which represents the communication link for the CAN-Bus and FlexRay-Bus, is examined. This results for the low frequency range to no differences. For the B-Band [8] the current in the twisted-pair-cables is twice as large than in the single wire. For that reason, as few wires as possible should be used.

A detailed specification of the measurements and the evaluation can be seen in [7].

Due to the results of the previous chapters, the following measurements are done with $45 \mathrm{kHz}$ PWM-frequency, $1000 \mathrm{rpm}$ and with full load. 


\section{Different shielding variants}

The components have been screened, before carrying out the measurements of the different shielding variants. The shielding of the components has a negligible influence on the interference, because the disturbance in the measured frequency range is a conducted interference. Figure 7 shows a part of the HV-cables with nearby communication cable and measuring clamp.

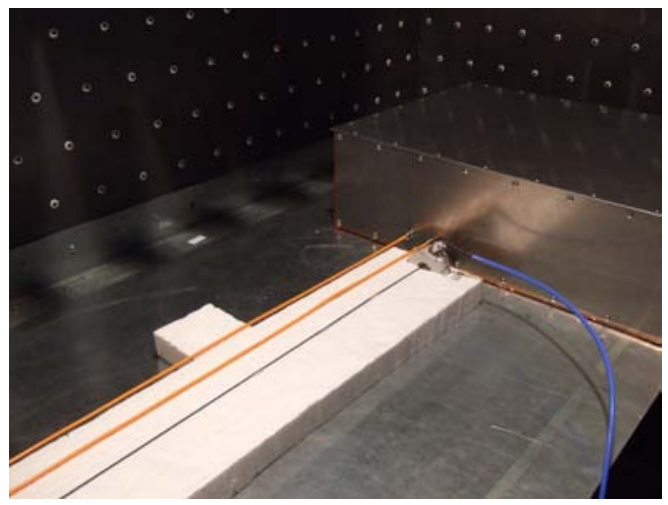

Fig. 7 HV-cables with nearby communication cable and measuring clamp

1) EMC-guarding-wire at each $H V$-cables / connected with one bridge

EMC-guarding-wire at the HV-cables minimize the EMC interferences. Therefore the EMC-guarding-wire is attached at both HV-cables in one case. The two EMCguarding-wires are connected in the middle with one wire, here called bridge, for the second case. A comparison of these two cases with the primary case with no shielding is shown in Figure 8. For the lower frequency range no differences are measured. The case with separated EMCguarding-wire on both $\mathrm{HV}$-cables leads to the best shielding effect. The connection between the two EMCguarding-wires leads to a lower degradation of the suppression due to enlarged ground loops.

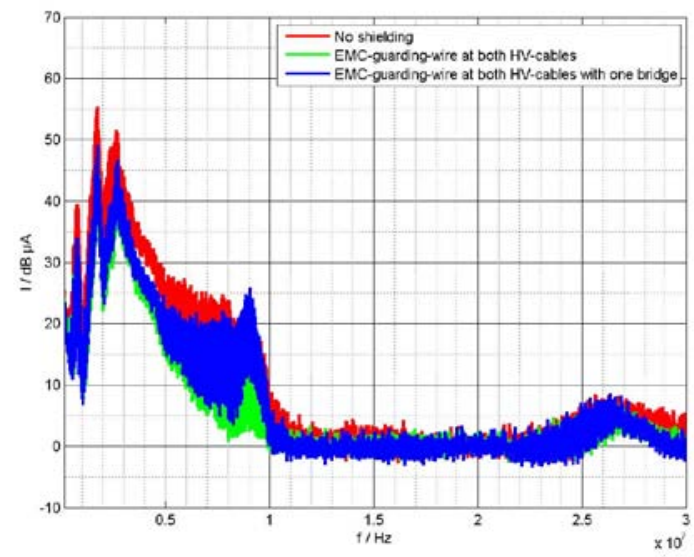

Fig. 8 Comparison of the conventional unshielded case, the case with EMC-guarding-wire at both $\mathrm{HV}$-cables and the case with EMC-guarding-wire at both HV-cables connected with one bridge
2) EMC-guarding-wire at the HV-cables with distributed brigdes / with spreaded connections

The intention of the distributed bridges layout is the minimization of the ground loops. This case is compared with the case of spreaded connections, where the bridges are spread out from the HV-cables parallel to the ground plane. The results are shown in Figure 9.

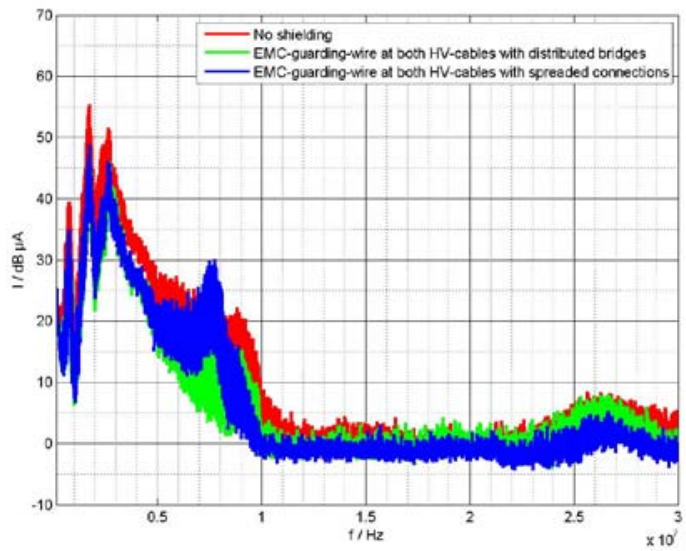

Fig. 9 Comparison of the conventional unshielded case, the case with EMC-guarding-wire at both $\mathrm{HV}$-cables with distributed bridges and the case with EMC-guarding-wire at both HVcables with spreaded connections

The spreaded connections show no improvement in the interference suppression. But it should be noted that the interference compared with the conventional unshielded case decreases. The smallest interference is shown for the distributed bridges case, but in comparison with the measurements before, the distributed brigdes delivers no big improvements. For the lower frequency range no differences are measured.

3) EMC-guarding-wire at the communication cable / at the HV-cables and at the communication cable

For the first case the EMC-guarding-wire is attached to the communication cable only. This is compared to the case with the EMC-guarding-wire at the communication cable and the HV-cables. No change is seen again for the A-Band range [8]. Figure 10 shows the results for the BBand. 


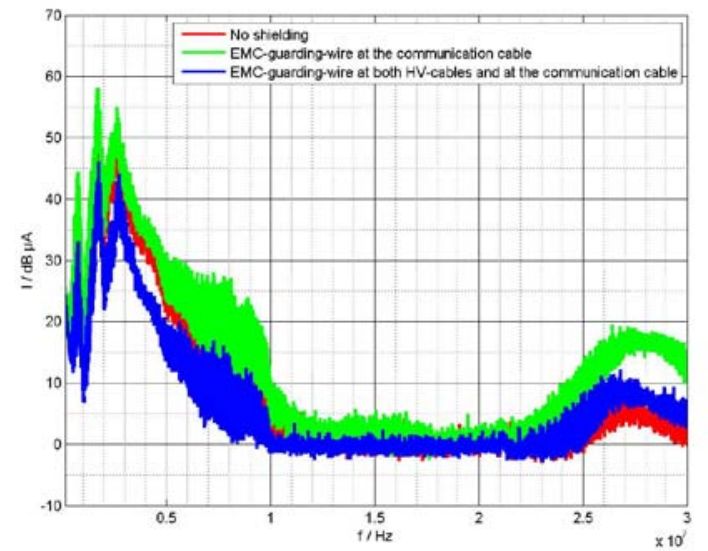

Fig. 10 Comparison of the conventional unshielded case, the case with EMC-guarding-wire at the communication cable and the case with EMC-guarding-wire at both $\mathrm{HV}$-cables and at the communication cable

One should think, the EMC-guarding-wire attached to the communication cable would bring a big improvement. But due to the vicinity of the measurement clamp and the EMC-guarding-wire the interference does not decrease compared to the conventional case. A much lower interference is seen for the attached EMC-guarding-wire to the HV-cables and the communication cables. The same interference behaviour can be seen in the case with EMCguarding-wires only at the HV-cables.

4) Comparison of the case with no shielding /with the best shielding variant / with full shielded HV-cables

Here the best shielding variant of the previous sections is compared with the full shielded HV-cables and with the conventional unshielded case. A picture of the shielded HV-cables connected to the housing is seen in Figure 11. Due to the connectors a good conductivity of the shield and the housing is given.

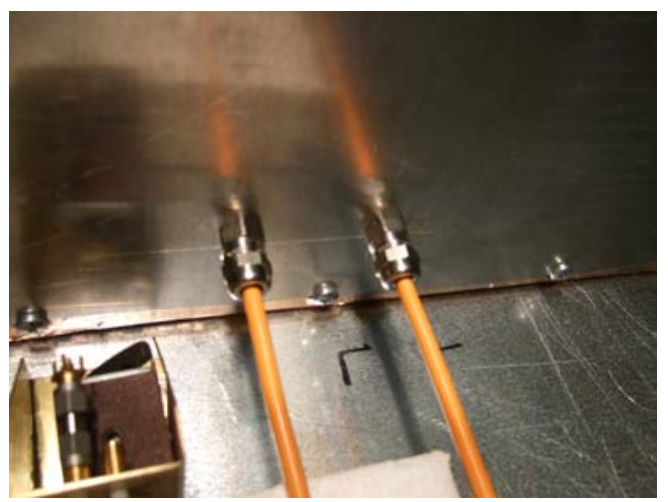

Fig. 11 Shielded HV-cables connected to the housing

For the frequency range from $9 \mathrm{kHz}-150 \mathrm{kHz}$ no differences are seen.

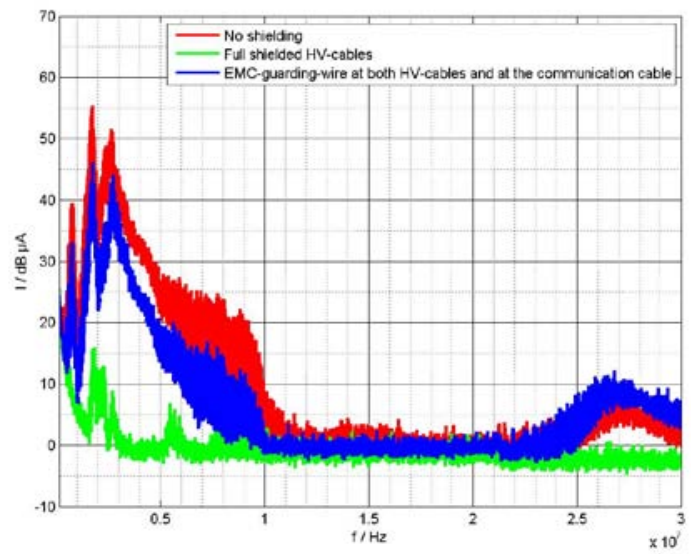

Fig. 12 Comparison of the conventional unshielded case, the case with full shielded HV-cables and the case with EMCguarding-wire at both $\mathrm{HV}$-cables and at the communication cable

Like it was expected, the full shielded case has the best shielding effect. It should be noted, that the best shielding variant decreased the interference by $10 \mathrm{~dB}$ and more, depending on the frequency range. With additionally meaningful positioning of the communication cables (see chapter B) further reduction of the interferences can be achieved.

\section{Representation of the chassis}

Not only different shielding variants can influence the interference, also the chassis and parts between the HVcables and the communication cable can change the interference behaviour. For that reason a metal plate is used to model the chassis in the transportation systems. Here the distance between the HV-cables and the communication cable is $30 \mathrm{~cm}$. The results are shown in Figure 13.

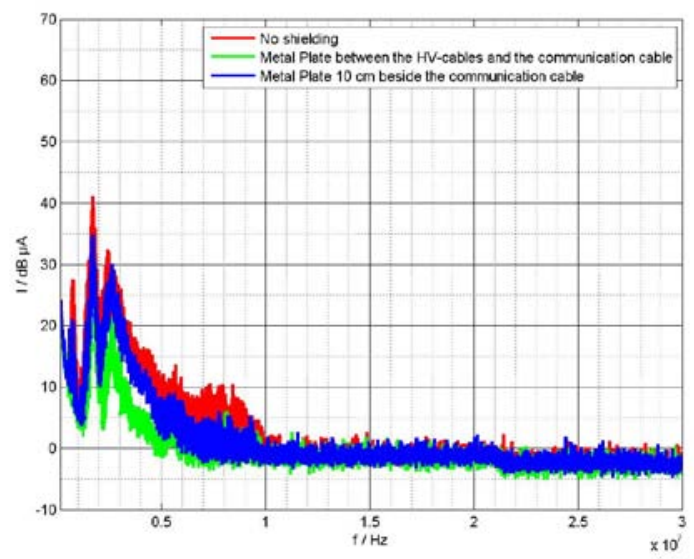

Fig. 13 Comparison of the conventional unshielded case, the case with a metal plate between the HV-cables and the communication cable and the case with a metal plate $10 \mathrm{~cm}$ beside the communication cable

For the frequency range from $9 \mathrm{kHz}-150 \mathrm{kHz}$ no differences are seen. The metal plate improved the shielding behaviour for the upper frequency range obviously. Conductive parts between the HV-cables and 
the measured cable improve the interference behaviour further.

The resulting currents can yield to load-voltages in Voltranges, depending on the terminations and on the voltage and current magnitude of the sources. For that reason, the interferences from the electrical drives to the communication systems should not be neglected, in order to avoid an influence up to a destruction of the communication.

\section{Conclusion}

In this paper an EMC study of an electrical powertrain for transportation systems is presented. There a summary of the interferences at the unshielded high-voltage cable (HVcable) is given. The analysis of the influence of position variation of the communication cable to the unshielded HV-cables is studied. Furthermore, the summary discussed the influence due to the change of parameters like the motor speed, the PWM-frequency, the terminations and the load. In addition, different cheap shielding variants are studied. Measurements and comparisons with full shielded $\mathrm{HV}$-cables as well as different optimizations to the test setup have also been realized.

Due to different positions of the communication cables to the HV-cables the results shown significant improvements. The different shielding variants delivered cheap opportunities to suppress the interferences.

To achieve a suppression of interferences on the communication lines, a meaningful positioning of communication cables with respect to HV-cables is encouraged. Using the knowledges gained in this study, the safety of the communication links can be increased and a higher security level and comfort thus obtained for the passengers.

\section{Acknowledgement}

The authors thank Mr. Helmut Kalb from Leoni AG for the material support provided for this study.

\section{References}

[1] C. C. Chan: The state of the art of electric and hybrid vehicles, Proceedings of the IEEE, 2002, pp. 247-275, doi:10.1109/5.989873, 2002

[2] J. M. Poinsignon, P. Matossian, B. Mazari, F. Duval: Automotive equipments EMC modeling for electrical network disturbances prediction, in Electromagnetic Compatibility, 2003. EMC '03.2003 IEEE International Symposium on, pp. 415-417, doi:10.1109/ICSMC2.2003.1428279, 2003

[3] M. Meyer and P. Asfaux: Radiated emissions modelling of a power cable, in Electromagnetic Compatibility, 2008. EMC Europe, 2008 International Symposium on, pp. 1-5, doi:10.1109/EMCEUROPE.2008.4786845, 2008

[4] S. Weber, E. Guttowski, E. Hoene, W. John and H. Reichl: EMC Issues in Cars with Electric Drives, in Electromagnetic Compatibility, 2003. EMC 2003. IEEE International Symposium, pp. 777-782, doi:10.1109/ISEMC.2003.1236706, 2003
[5] A. F. Moreira, T.A. Lipo, G. Venkataramanan and S. Bernet: High frequency modeling for cable and induction motor overvoltage studies in long cable drives, Industry Applications Conference, 2001. Thirty-Sixth IAS Annual Meeting. Conference Record of the 2001 IEEE, pp. 1787-1794, doi:10.1109/IAS.2001.955774, 2001

[6] A. Kosonen and J. Ahola: Comparison of signal coupling methods for power line communication between a motor and an inverter, 2009 IET 2009. IET Electric Power Applications, pp. 431-440, doi:10.1049/iet-epa.2009.0114, 2009

[7] B. Chand, K. Hähre, J. Keghie and S. Dickmann: An EMC study of an electrical powertrain for transportation systems (accepted for publication in URSI Advances in Radio Science)

[8] CISPR 16-2-1: Specification for radio disturbance and immunity measuring apparatus and methods Part 2-1: Methods of measurement of disturbances and immunity - Conducted disturbance measurements, 2008 